\title{
Mathematical approach to human emotion processing
}

\author{
2020/08/30 First Edition 2020/11/18 15th Edition \\ Takuya Yabu(takuya.yabu@live.jp)
}

\begin{abstract}
When I belonged to a group, I was always bullied no matter how I behaved, so I didn't know how to handle my own emotions, so I considered human emotions. I mathematically modeled human emotion processing in two stages: what kind of emotions we receive from events and how we react from the emotions we receive. The part that receives emotions from events and the part that responds from emotions are modeled by a one-dimensional random walk or Wiener process, and the distribution of individual emotions is represented by a fixed probability distribution, and the response of individual is represented by a fixed distribution. Therefore, I also showed that when individuals gather to form a group, the distribution of emotions and reactions as a group is also represented by a fixed distribution. In addition, I showed as application examples of these models, the nature of events, the meaning of emotional distribution, and how to read the air, and so on.
\end{abstract}

\section{Introduction}

When I belong to some group, even if I act like myself, or if I suppress myself and act to apologize as soon as I think I did something wrong, there are only patterns of being bullied and isolated. I don't even know how to react to the feelings from. Also, I am often anxious, and I am not sure how to deal with anxiety and how to react to my emotions.

Therefore, I thought about how human emotions are processed and how I can deal with it because I can't handle it well.

2. About personal emotion processing

2.1. About individual reaction functions

Suppose that when an event $X$ occurs, the emotion $x$ occurs. In response to this, a reaction called $F(x)$ occurs. Figure 1 shows the relationship.

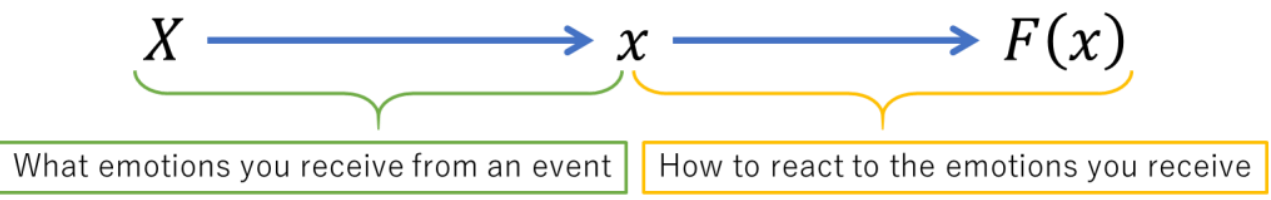

Figure 1 About the relationship between events, emotions, and reactions 
Here, human beings cannot judge whether an event is emotionally good or bad immediately after it occurs. Therefore, the emotion $x$ in that case is 0. From there, determine whether it is good or bad. In that case, suppose you enumerate a certain number of times $n$, such as good on one side and bad on another side. If you enumerate the good things $k$ times $(0 \leq k \leq n)$, the combination is given by the following equation.

$$
{ }_{n} C_{k}
$$

Here, for the sake of simplicity, if you decide that this part is a good point in the event, add 1 to emotion $x$, and if you decide that this part is a bad point, add -1 . Then, when emotion $x$ is $k=\frac{n}{2}$, that is, when both good and bad are the same number $x=0$, the equation ( 1 ), which is the number of combinations, is maximized.

In other words, we think that emotion processing is performed by a one-dimensional random walk. With a probability of $\frac{1}{2}$, it is judged whether it is a positive emotion or a negative emotion, and it is considered that the total emotion is obtained by adding them together. At this time, from the one-dimensional random walk, the mean (expected value) $\mu$ of emotion $x$ is 0 , and the variance $\sigma^{2}$ is $n$.

Now, suppose that this emotion processing is performed $m$ times. At this time, the value of each emotion is $x_{i}(i=1, \cdots, m)$. From the central limit theorem, the following holds for a certain probability distribution $P$.

$$
\begin{gathered}
\lim _{m \rightarrow \infty} P\left(\frac{x_{1}+\cdots+x_{m}-m \mu}{\sqrt{m} \sigma} \leq \alpha\right)=\lim _{m \rightarrow \infty} P\left(\frac{\frac{x_{1}+\cdots+x_{m}}{m}-\mu}{\frac{\sigma}{\sqrt{m}}} \leq \alpha\right) \\
=\frac{1}{\sqrt{2 \pi}} \int_{-\infty}^{\alpha} e^{-\frac{x^{2}}{2}} d x
\end{gathered}
$$

Now consider $\sigma^{2} \cdot \sigma^{2}=n_{1}+\cdots+n_{m}$, but it is thought that the value is dominated by the largest $n$. Therefore, when $n \rightarrow \infty$ exists, $\sigma^{2} \rightarrow n$. Also, this time $\mu=0$. Therefore,

$$
\lim _{m, n \rightarrow \infty} P\left(\frac{\frac{x_{1}+\cdots+x_{m}}{m}}{\frac{\sqrt{n}}{\sqrt{m}}} \leq \alpha\right)=\frac{1}{\sqrt{2 \pi}} \int_{-\infty}^{\alpha} e^{-\frac{x^{2}}{2}} d x
$$

Therefore, when $m, n \rightarrow \infty$, it can be regarded as $n \rightarrow m$, and the denominator $\frac{\sqrt{n}}{\sqrt{m}}$ approaches 1 from equation (3), so the following equation holds.

$$
\lim _{m, n \rightarrow \infty} P\left(\frac{x_{1}+\cdots+x_{m}}{m} \leq \alpha\right)=\frac{1}{\sqrt{2 \pi}} \int_{-\infty}^{\alpha} e^{-\frac{x^{2}}{2}} d x
$$

That is, the probability distribution of emotions' mean converges to the standard normal 
distribution. Therefore, the probability density function $p(x)$ of emotions' mean is expressed by the following equation.

$$
p(x)=\frac{1}{\sqrt{2 \pi}} e^{-\frac{x^{2}}{2}}
$$

The probability density function of an individual's emotions can be expressed by equation ( 5 ) if the emotion processing is repeated many times and they are continuously averaged. Based on this function, the individual is performing emotional processing. Considering that the higher the probability, the larger the reaction, the reaction function $F(x)$ is expressed by the following equation using a certain positive constant $q_{0}$.

$$
F(x)=\frac{q_{0}}{\sqrt{2 \pi}} e^{-\frac{x^{2}}{2}}
$$

This formula determines the reaction based on the emotion $x$ received for the event $X$ that occurred.

2.2. About the reaction function obtained from the one-dimensional random walk

The discussion so far can also be found from the one-dimensional random walk. Think from this point of view. The probability density function for a one-dimensional random walk is given by the following equation.

$$
p(x, n)=\frac{1}{\sqrt{2 \pi n}} e^{-\frac{x^{2}}{2 n}}
$$

Therefore, the reaction function is given by the following equation.

$$
F(x, n)=\frac{q_{0}}{\sqrt{2 \pi n}} e^{-\frac{x^{2}}{2 n}}
$$

For equation ( 7), consider the variance of $x$. Assuming that emotion processing is performed $\mathrm{m}$ times, let $x_{j}(j=1, \cdots, m)$ and consider the following formula. Note that the average of $x_{j}$ is 0 here. Here, $\hat{\sigma}^{2}$ is an unbiased variance.

$$
\tilde{\chi}^{2}=\frac{x_{1}^{2}}{\frac{\hat{\sigma}^{2}}{(m-1)}}+\cdots+\frac{x_{m}^{2}}{\frac{\hat{\sigma}^{2}}{(m-1)}}=\frac{1}{\frac{\hat{\sigma}^{2}}{(m-1)}} \sum_{j=1}^{m} x_{j}^{2}
$$

In addition, the formula transformation continues.

$$
\tilde{\chi}^{2}=\frac{1}{\hat{\sigma}^{2}} \sum_{j=1}^{m} x_{j}^{2}=\frac{\frac{1}{\frac{\sigma^{2}}{(m-1)}} \sum_{j=1}^{m} x_{j}^{2}}{\frac{1}{(m-1)} \cdot \frac{(m-1) \hat{\sigma}^{2}}{\sigma^{2}}}
$$


That is, $\frac{\tilde{\chi}^{2}}{m-1}$ is an $F$ distribution with two degrees of freedom $m-1$.

Let us consider $\frac{\tilde{\chi}^{2}}{m-1}$ again from equation (9). When the number of times emotions are processed $m \rightarrow \infty$, the following formula holds.

$$
\begin{aligned}
& \frac{\tilde{\chi}^{2}}{m-1}=\frac{1}{m-1}\left(\frac{x_{1}^{2}}{\frac{\hat{\sigma}^{2}}{(m-1)}}+\cdots+\frac{x_{m}^{2}}{\frac{\hat{\sigma}^{2}}{(m-1)}}\right) \\
& =\frac{1}{m-1}\left(\frac{x_{1}^{2}}{\frac{(m-1)}{(m-1)}}+\cdots+\frac{x_{m}^{2}}{\frac{(m-1)}{(m-1)}}\right) \rightarrow \frac{x_{1}^{2}+\cdots+x_{m}^{2}}{m}
\end{aligned}
$$

In other words, $\frac{\tilde{\chi}^{2}}{m-1}$ is the variance of emotion $x$. Therefore, the variance $\sigma^{2}$ follows an $F$ distribution with two degrees of freedom (number of emotional processes) of $m-1$.

Next, consider the reaction function. The mean of the reaction functions is 0 and the variance is $q_{0}^{2} \sigma^{2}$. Consider the following variables, where the reaction value is $y_{j}(j=$ $1, \cdots, m)$.

$$
\widetilde{\Upsilon}^{2}=\frac{y_{1}^{2}}{\frac{\hat{\sigma}^{2}}{(m-1)}}+\cdots+\frac{y_{m}^{2}}{\frac{\hat{\sigma}^{2}}{(m-1)}}
$$

That is, similar to the discussion of equations ( 9) to ( 11$), q_{0}^{2} \sigma^{2}$ follows an $F$ distribution with two degrees of freedom $m-1$.

Therefore, $q_{0}^{2}$ also follows the $F$ distribution with two degrees of freedom $m-1$, considering from the following equation.

$$
q_{0}=\frac{q_{0} \sigma^{2}}{\sigma^{2}}=\frac{\frac{\tilde{\chi}^{2}}{m-1}}{\frac{\widetilde{\Upsilon}^{2}}{m-1}}
$$

The graph of $\sigma^{2}, q_{0}^{2} \sigma^{2}, q_{0}^{2}$ is as follows. All have an $F$ distribution with two degrees of freedom (number of emotional processes) of $m-1$. 


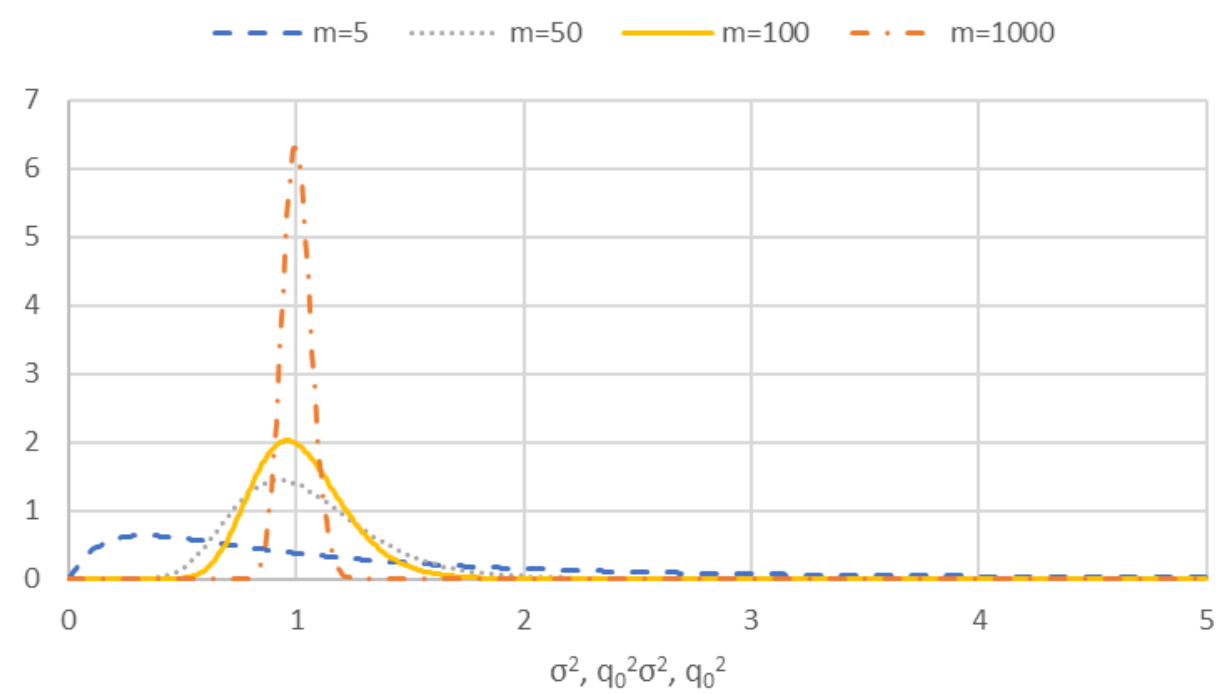

Figure 2 Graph of $\sigma^{2}, q_{0}^{2} \sigma^{2}, q_{0}^{2}$ ( $F$ distribution with two degrees of freedom $m-1$ )

The mean and variance of the $F$ distribution with two degrees of freedom $m-1$ are:

$$
\text { Mean } \frac{m-1}{m-3} \text {, Variance } \frac{4(m-1)(m-2)}{(m-3)^{2}(m-5)}
$$

In other words, when $m \rightarrow \infty$, the mean is 1 and the variance is 0 . This is consistent with the variance $\sigma^{2}=1$ in the probability distribution of emotions' mean in Section 2.1. Also, $q_{0} \rightarrow 1$. In other words, the individual reaction function is given by the following formula.

$$
F(x)=\frac{1}{\sqrt{2 \pi}} e^{-\frac{x^{2}}{2}}
$$

\subsection{From discrete to continuous}

Regarding the above, replace "listing $n$ times" with thinking for a certain period of time. Therefore, consider the following Wiener process. Here, $\beta$ is a positive real number and $W_{t}$ is a standard Brownian motion. $\beta$ is your whimsical ratio.

$$
x=\beta W_{t}
$$

Now, from equation ( 16), which is a stochastic differential equation, the following equation can be obtained. This is an equation called the Fokker-Planck equation. Where $p(x, t)$ is the probability density function.

$$
\frac{\partial p}{\partial t}=\frac{1}{2} \beta^{2} \frac{\partial^{2} p}{\partial x^{2}}
$$

Consider also from equation ( 1$)$. Let $P(x)$ be the probability of taking a certain emotion $x$. Now, suppose that emotions change by a small amount $a$. At this time, 
consider the following values

$$
-\{P(x+a)-P(x)\}
$$

This value represents the amount of change in the probability that emotions change, and is also the amount required to change a minute amount $a$. Consider the following formula as an amount considering how much it changes per unit time $\Delta t$.

$$
Q=-\frac{1}{\Delta t}\{P(x+a)-P(x)\}=-\frac{1}{\Delta t} \frac{d P}{d x} a
$$

This $Q$ represents the amount required to change a minute amount a per unit time.

From equation (19), the following equation is obtained with $p(x)=\frac{P(x)}{\mathrm{a}}$.

$$
Q=-\frac{a^{2}}{\Delta t} \frac{d p}{d x}
$$

Now, the idea in equation ( 1 ) is the same as the one-dimensional random walk. Here, considering how much $a$ is a value per unit amount of $x$, it is 2 because it takes a positive value and a negative value. That is, $a=\frac{1}{2}$. Also, since $\Delta t$ needs to obtain a for two times, this is also $\Delta t=\frac{1}{2}$. Therefore, the following equation can be obtained by substituting it into equation ( 20 ).

$$
Q=-\frac{1}{2} \frac{d p}{d x}
$$

Consider the time change of this $p$. The magnitude of $p$ per minute amount $d x$ of an emotion is $p d x$. Considering the amount of change over time, it is $\frac{\partial p}{\partial t} d x$. Considering how much $Q$ changes with respect to this $d x$, the following equation is obtained.

$$
Q(x)-Q(x+d x)=\frac{1}{2} \frac{\partial^{2} p}{\partial x^{2}} d x
$$

This value is equal to $\frac{\partial p}{\partial t} d x$. Therefore, the following partial differential equation is obtained.

$$
\frac{\partial p}{\partial t}=\frac{1}{2} \frac{\partial^{2} p}{\partial x^{2}}
$$

The partial differential equation (23) obtained from equation (1) and the Fokker-Planck equation (17) are the same equation. So you get:

$$
\beta^{2}=1, \beta=1
$$

This is the formula that $\beta$ satisfies.

From equation ( 23), the following equation is obtained. Here, $\mathrm{t}$ is the time until the emotion processing is completed. 


$$
p(x, t)=\frac{1}{\sqrt{2 \pi t}} e^{-\frac{x^{2}}{2 t}}
$$

The reaction function $F(x)$ is expressed by the following function, which is obtained by multiplying the probability density function of equation (25) by the proportional value $q_{0}$.

$$
F(x, t)=\frac{q_{0}}{\sqrt{2 \pi t}} e^{-\frac{x^{2}}{2 t}}
$$

From the discussion so far, the average distribution of emotions after many emotional processes is 0 on average and 1 on variance. Therefore, in equations ( 25) and (26), $t \rightarrow 1, q_{0} \rightarrow 1$.

3. About emotional processing when forming a group

3.1. About the reaction function of the group

Consider when individuals are grouped together. We consider the emotional processing of a group to be the average of the group. Then, of course, the probability density function of the group's emotions is equal to equation ( 5 ) and is given by:

$$
p_{\text {group }}(x)=\frac{1}{\sqrt{2 \pi}} e^{-\frac{x^{2}}{2}}
$$

Also, the reaction function is equal to equation (15) and is given by:

$$
F_{\text {group }}(x)=\frac{1}{\sqrt{2 \pi}} e^{-\frac{x^{2}}{2}}
$$

The graph of equation (28) is as follows.

- Reaction Function

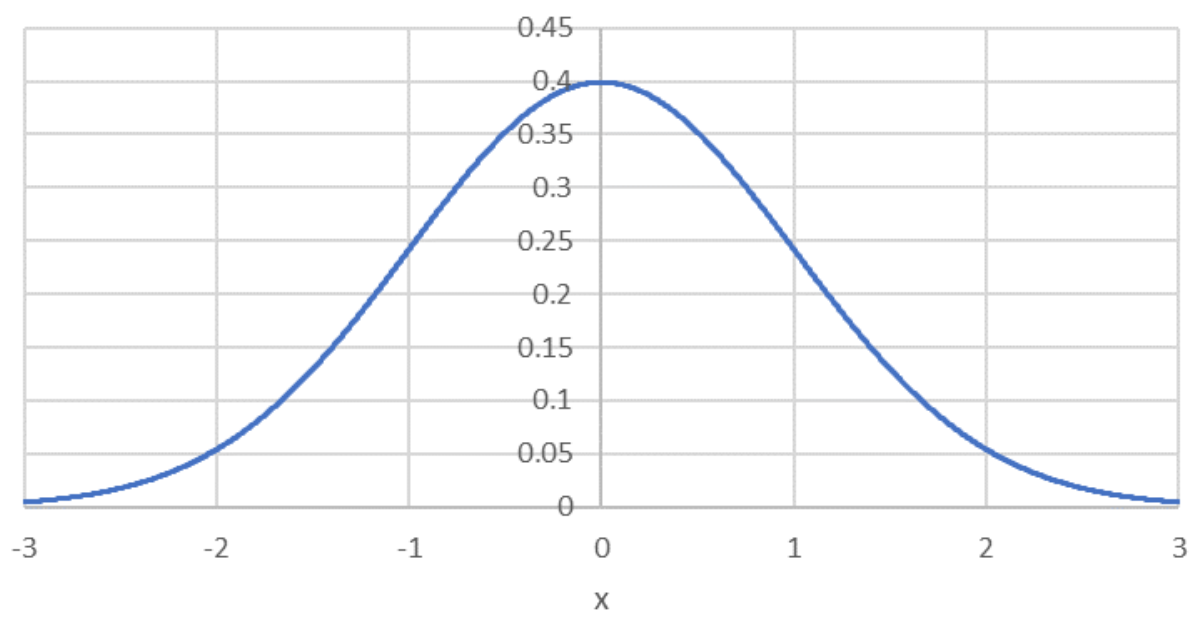

Figure 3 Individual and group reaction function 
From Figure 3, it can be seen that the response function of the group's mean produces a wide range of emotions. It turns out that when humans are grouped together, they perform this kind of emotional processing.

\subsection{About emotion processing in a group}

From Section 3.1, the reaction function of the group is expressed by an equation.

$$
F_{\text {group }}(x)=\frac{1}{\sqrt{2 \pi}} e^{-\frac{x^{2}}{2}}
$$

We also found that the individual reaction function is equation (15).

From these results, we can see that the reaction functions of individuals and groups are equal. From this, it can be said that various things can be said. First, you don't have to think about your own boundaries. Since every individual has the same emotional probability density function and reaction function, What I hate is what the group hate, and What I like is what the group like. The only difference is whether you can read the air well or not. If you don't like it, you can say you don't like it.

Also, democracy is established as a political means because it is easy to select people who can read the air by majority vote, and since such representatives have the same reaction function, they will execute the desired policy. In a tyranny, it is possible that a person who does not know how to read the air will become king due to his pedigree, and if that happens, it will not work well.

Furthermore, it can be said that a group functions as a group because the reaction functions of individuals and groups are equations (29) and (15). When many people get together, the reaction function is the same without doing anything, so control can be taken as a group. Therefore, people who do not know how to read the air stand out. They don't get used to the group. Therefore, it is thought that the problem of bullying and power harassment will occur if people who do not understand how to read the air are divided into those who attack and those who are attacked. On the other hand, most humans are indifferent because they do not know how to react because the reaction from people who do not understand how to read the air is different, or because they do not react because they do not disturb the group. Considering that the probability of not knowing how to read the air is small, it can be explained that bullying and power harassment occur in small groups. It is also possible that the attacker wants to atrophy most humans by becoming aggressive because he is not eliminated.

When three people get together, they may be divided into two and one, probably because there are two people who can read the air and one is a little weak at reading the air. 
I wrote that I don't know how to read the air, but I have the same emotional probability density function as the neurotypical one, and the reaction function is also the same. In other words, it's just a small difference. Because the reaction of the person who are attacked is interesting to the person who attack, bullying and power harassment will probably occur.

3.3. What does the value of the reaction function mean?

Now let's consider the value of the reaction function.

As shown in Figure 1, it receives emotions and reacts to events. Events include events from the natural world, but in most cases we receive reactions from others as events.

In other words, the reaction of others becomes an event as it is. That is, the following relational expression is considered to hold.

$$
X=F(x)
$$

Using equation (30), it is possible to find the emotion $x$ from the event $X$. It is very inefficient to ask humans to receive emotions from events from a one-dimensional random walk. They are tired. Therefore, in most cases, it is considered that the emotion $x$ is calculated from equation ( 30 ). However, from Figure 3, from one event $X$, emotion $x$ takes either a positive or negative value.

Whether to take a positive or negative value differs depending on whether the event $X$ is received from an entity that has emotion $x$, such as an individual or a group, or if the event $X$ is received from an entity that does not have emotion $x$, such as the weather.

First, when we receive event $X\left(0 \leq X \leq \frac{1}{\sqrt{2 \pi}}\right)$ from an entity that has emotion $x$. In this case, the emotion is calculated directly from equation ( 30 ). At that time, the positive or negative is determined by the individual's tendency. Even if you receive the same event, depending on the individual, it may be considered positive or negative.

Next, when event $X$ is received from an entity that has no emotions such as the weather, in this case as well, the emotions are calculated directly from equation (30). At that time, the positive and negative are also determined by the individual's tendency. It's the same as some people who are happy and some who slap their tongue when it rains.

Now, from Figure 3, we can see that emotion $x$ has no solution when $X>\frac{1}{\sqrt{2 \pi}}(\approx 0.4)$. Individuals cannot have emotions in events that give a great reaction. In this case, we think that we will deal with it as follows.

1. Ignore event $X$. 
2. Reject event $X$.

3. In an attempt to understand event $X$, we try to obtain emotion $x$ using equation (16).

Especially think about 3 . If you take the time to get emotion $x$, you get tired. At this time, your own reaction $X$ becomes smaller. On the other hand, if the brain automatically gives emotion $x$ from equation ( 30 ), as can be seen from Figure 3, emotion $x$ has a large positive value or a large negative value. Since we spend a lot of time thinking about big reactions, we usually choose big negative values, which can show big negative emotions, extreme disgust, extreme anger, or extreme disappointment.

If event $X$ with a big reaction is brought about by the action of one person, for example, like me, it is quite a problem if it makes a big reaction with a little emotion. For example, if the value of the reaction function is confused and a large reaction is made many times with a slight negative emotion, the sum of the event values is $\frac{1}{\sqrt{2 \pi}}$ as shown in Section 3.4 will exceed. When that happens, the other person becomes extremely disgusted or extremely angry after being seriously wondering whether to ignore it, reject it, or "why do you react so big?" For example, if you say "I'm sorry, I'm sorry" in a very loud voice, it's just a trivial action for the other party. So if you give an unexpectedly big reaction from it, you will not get a good reaction from other party. Therefore, they are ignored or rejected by the group. Also, if the same behavior continues frequently, the person who is worried with him will judge that he is "a person who can be seen below to that extent" from his extreme disgust, anger, and disappointment. This does not make human relationships smooth. It causes bullying and power harassment.

Also, consider the case where event $X$ with a large reaction is given. For example, consider being given a job that cannot be handled many times, or being given inhumane words and actions many times. In this case, as shown in Section 3.4, the sum of the event values exceeds $\frac{1}{\sqrt{2 \pi}}$, so it is okay for individuals who can ignore or reject, such as 1 and 2 , but as in 3 . If you worry, and if you worry for a long time, you will get tired. If you make a small reaction and the brain automatically gives emotion $x$ to it from equation (30), you feel great negative feelings such as extreme self-loathing, extreme anger at yourself, or extreme disappointed in yourself. If it continues over and over again, you will end up with depression in the worst case, with extreme self-loathing, anger at yourself, and disappointment in yourself. In this case, it is important to be quick and free from the environment where event $X$ is given many times. 
This model can also explain that you aren't valued when you help someone else's work or do chores at your company. At first, the reaction value of the event is small because the other party also asks for small things. Therefore, a big positive emotion $x$ is generated, "I am nice to help", and the other party also has a big positive emotion and "helped", but if that continues, the reaction value of the event will accumulate, as shown in Section 3.4. As you can see from Figure 3, the positive value of emotions becomes smaller, and it becomes more natural to say, "You will do it this time as well. Because you are kind of existence." So it's a good idea to show big negative emotions while showing the first small reaction value and say, "Your job? I don't do that."

Also, the sooner you get the results of your work, the smaller the expectation value of the other party, that is, the event with a small reaction. So as you can see from Figure 3, the positive emotions increase, so the other party say "It's early. Thank you." Therefore, even a small mistake is likely to be tolerated. On the contrary, if you submit it over time, it will be an event with a large value as a reaction. So it will be "because it took time", and at the same time the positive emotions will be reduced, the degree of perfection will be required, and the other party say "Even though you took time, there is a mistake". There is a possibility that the positive feelings will become smaller. I think it is good for you and the other party not to make the size of the event so large, such as submitting it as soon as possible.

I think the same thing can be said about the expected value of the other party. If you expect a big reaction, "because you are on good terms with that person" and "because that person will do this much for other people", after receiving it, the reaction is big as you can see from Figure 3. Since it is regarded as a big event, it becomes "this level? I'm not so happy". Instead, you can get a big positive feeling by making a small reaction so that you can recognize "you did this even though I did not expect it". If you want to spend your days in peace, I think it's best to live in neutral, with as few expectations as possible.

Finally, about the value of the event. The maximum value is $\frac{1}{\sqrt{2 \pi}}$ when $x=0$, but it is in a safe and stable state at this time. From the outside, it looks like nothing is happening, but the value of the event at this time is the maximum value. If you take any action from this state, the value of the event will decrease. When you are doing big things from the outside, your emotions are big positive and negative values. In other words, the value of the event is small. In general, during intense behavior, the reaction function, or event value, is small. You need to be careful about this point. 


\subsection{About the characteristics of the event}

Consider the characteristics of event $X$.

Events become smaller and forgotten over time. It is also likely to be forgotten or remembered. Therefore, consider a one-dimensional random walk with the value $\tau$ (unit is seconds, but 0 if it is 0 or less) minus the period judged to be important from the period judged to be insignificant. Derived using the central limit theorem and so on, the probability density function can be obtained by the following equation.

$$
p(\tau)=\sqrt{\frac{2}{\pi}} e^{-\frac{\tau^{2}}{2}}, \tau \geq 0
$$

Therefore, for events, using equation (31), think in the same way as equation (6), and obtain the following equation as a function of the event considering the initial value of the event.

$$
X=X_{0} e^{-\frac{\tau^{2}}{2}}, \tau \geq 0
$$

Where $X_{0}$ represents the initial value of the event.

That is, most individuals, and groups of individuals, forget about event $X$ in equation (32).

Also consider that event $X$ is repeated $n$ times. In this case, the following formula holds.

$$
X=X_{0} e^{-\frac{\tau_{1}^{2}}{2}}+X_{0} e^{-\frac{\tau_{2}^{2}}{2}}+\cdots+X_{0} e^{-\frac{\tau_{n}^{2}}{2}}=n X_{0} e^{-\frac{\tau_{1}^{2}+\cdots+\tau_{n}^{2}}{2 n}}
$$

In other words, the larger $n$, the larger the value of the event and the harder it is to forget.

From this, if you repeat the same action or thinking about something, the value of event $X$ will increase in individuals or groups of individuals, and it will exceed $\frac{1}{\sqrt{2 \pi}}$, resulting in rejection or a big negative to yourself. As mentioned in Section 6, you can think unconsciously as many times as you like in one second. If you give the same actions and thoughts, it is better to open the period and reduce the number of times as much as possible. Repeated actions and thoughts can lead to resentment and depression. Even good behavior can be disgusting and rejected if you repeat it over and over again.

\subsection{Tips for worrying}

Human's $\beta$ is 1 , and the value does not change. And, due to the nature of the $F$ distribution, $t \rightarrow 1$. First, regardless of whether you are worried for a long time or for a 
short time, the reaction function of an individual does not change in equation (15), and the shape of the graph does not change from that given in Figure 3. Even if you worry for a long time, your emotional range will not expand. Even if you worry for a short time, your emotional range will not be narrowed. The stochastic process expressed by equation ( 16 ) is called the Ornstein-Uhlenbeck process (mean reversion process), and when the time $t$ to worry becomes longer, the emotion $x$ approaches the mean value 0 . In other words, the more you worry, the less positive and negative emotions you get. It makes you feel emotionless and very tired.

In other words, even if you worry for a long time, you will get tired, and if you make a small reaction and the brain automatically gives emotion $x$ to it from equation (30), you feel great negative feelings such as extreme self-loathing, extreme anger at yourself, or extreme disappointed in yourself. There is nothing good about it.

Therefore, if you are worried, I think it is better to set a short time and worry only for that time.

4. What to do if you don't get along well with your surroundings

4.1. About reaction function

The probability density function and reaction function of an individual's emotions are the same for everyone. However, you may be wondering how happy or sad the reaction function is, how happy or sad others are, and what the maximum value is. me too.

First, about happiness and sadness. The response functions of individuals and groups in Figure 3 are 34\% when emotion $x$ is between 0 and 1 (between emotionlessness, peace of mind, stability and normal happiness), 14\% when emotion $x$ is between 1 and 2 (very happy), and $2 \%$ when 2 or more(very, very happy), includes happy feelings. It is $50 \%$ in total. If it is $100 \%$, it will be doubled.

Think about this in a year. Most people feel "very, very happy" with the joy that occurs for about 14 days, which is $4 \%$ of 365 days. This is about once a month, which is about a payday. In addition, most people feel "very happy" with the joy that occurs for about 102 days, which is $28 \%$. This is the joy of having about two days a week, like giving something to yourself on Saturday and Sunday. What you feel on other days is "from emotionlessness, peace of mind, stability to normal joy." Negative emotions are the opposite of this.

The degree of joy and sadness is as above. Now, let's consider how much the maximum value of the reaction function is.

I think that emotion $x=0$, that is, the value when reacting without thinking in a state of complete peace of mind is the maximum value. I think this is a reaction that wakes 
up in the morning and greets parents and other family members with a carefree "good morning". At times like this, there aren't many people who cheerfully say "Good morning!" I think this is the maximum value.

These ideas can be said from the reaction functions of individuals and groups. I will try it too. I will report any progress.

\subsection{How to read the air}

If you don't get along well with your surroundings, you may not be able to know what happened or what the other person was doing. It means that you cannot read the so-called air.

So, let me give you an idea of how to read the air. First, we try to find out from the emotion processing itself. Here, the flux of emotion processing is defined by the following formula. Same as equation (21).

$$
Q=-\frac{1}{2} \frac{\partial p}{\partial x}=-\frac{1}{2} \frac{\partial F}{\partial x}
$$

Now, regarding the flux $Q_{y}$ of the emotion processing of the other party given by equation (34), if the event is dimensionless and the emotion has a dimension of $\mathrm{E}$, the dimension of $Q_{y}$ is $\left[\mathrm{ET}^{-1}\right]$. Note that $Q_{y}=\frac{d y}{d t}$ cannot be used here. $x$ is a random variable, and $Q_{y}=\frac{d W_{t}}{d t}$ from equation (16), but there is no time derivative of the Wiener process. Therefore, it is the emotional value per unit time, such as $Q_{y}=\frac{y}{\Delta t}$. If you know $Q_{y}$, you can understand the feelings of the other person. For this reason, I want to measure $Q_{y}$, but I need to know the emotions of the other person in order to do it directly, but I do not know that. Therefore, it is calculated from $Q_{y}=-\frac{1}{2} \frac{\partial F}{\partial y} . \partial F$, that is, $d F$, can be understood by looking at the change in the reaction of the other party. However, I do not know $\partial y$, that is, $d y$, the minute change of the other person's emotions. Therefore, the flux $Q_{y}$ of the emotion processing of the other party cannot be measured directly or indirectly.

Therefore, another method is used to obtain the emotional value of the other party. The graph in Figure 3 shows the reaction function of most individuals and groups. Take advantage of this.

First, slightly increase the value of yourself and the reaction function to $d F$. Since the other party also increases slightly with $d F$, if the other party's emotion has a positive sign, the emotional minute change $d y$ tends to decrease, and if the other party's 
emotion has a negative sign, the emotional minute change $d y$ tends to increase. From this result, the reaction from the other party is received with a slight increase of $d F$. At this time, the minute change $d x$ of one's emotions for changing one's reaction also changes in the same way as $d y$.

Next, reduce the value of yourself and the reaction function to $d F$. Since the other party also decreases slightly with $d F$, if the other party's emotion has a positive sign, the emotional minute change $d y$ tends to increase, and if the other party's emotion has a negative sign, the emotional minute change $d y$ tends to decrease. From this result, the reaction from the other party is received with a slight decrease of $d F$. At this time as well, the minute change $d x$ of one's emotions for changing one's reaction changes in the same way as $d y$.

In other words, the sign of the other person's emotions can be understood from the change in the other person's behavior immediately after the person's reaction is slightly increased or slightly decreased. Immediately after you have a positive emotion and slightly increase your reaction and slightly decrease your emotion, if the other person reacts with a negative emotional change, the other person and yourself have a positive emotion. Or immediately after you have a positive emotion and slightly decrease your reaction and slightly increase your emotion, if the other person reacts with a positive emotional change, also the other person and yourself have a positive emotion. Immediately after you have a negative emotion and slightly increase your reaction and slightly increase your emotion, if the other person reacts with a positive emotional change, the other person and yourself have a negative emotion. Or immediately after you have a negative emotion and slightly decrease your reaction and slightly decrease your emotion, if the other person reacts with a negative emotional change, also the other person and yourself have a negative emotion.

On the contrary, immediately after you have a positive emotion and slightly increase your reaction and slightly decrease your emotion, if the other person reacts with a positive emotional change, the other person has a negative emotion. Or immediately after you have a positive emotion and slightly decrease your reaction and slightly increase your emotion, if the other person reacts with a negative emotional change, also the other person has a negative emotion. Immediately after you have a negative emotion and slightly increase your reaction and slightly increase your emotion, if the other person reacts with a negative emotional change, the other person has a positive emotion. Or immediately after you have a negative emotion and slightly decrease your reaction and slightly decrease your emotion, if the other person reacts with a positive emotional change, also the other person has a positive emotion. 
As an example, suppose you are talking about something with positive emotions. By stopping the conversation or slowing down the pace of the conversation, your reaction will be slightly increased (your emotions will be slightly decreased). Immediately after this, if the other person makes a boring negative emotional change such as a boring expression, it can be judged that the other person also has a positive emotion with the same sign and wants to hear the story. On the other hand, if the other person makes a confused facial expression immediately after a slight decrease in one's reaction (a slight increase in one's emotions), such as increasing the pace of conversation, the other person makes a different sign You can judge that you do not want to hear the story because of your negative emotions.

"Reading the air" does not mean that there is any special number, but it is obtained by changing one's reaction and seeing the change in the behavior of the other party. People with high-functioning autism, such as Asperger's Syndrome, just didn't know how to do it.

If you can read the air, that is, know the sign of the other person's emotions, you can also know the value of the other person's emotions. From equation (30), the absolute value of the other person's and one's emotions is the same because the reaction function is the same. Therefore, if the same sign is used, the other person's feelings are the same, and if the signs are different, the other person's feelings are the same size and opposite. I have been bullied no matter what group I belong to. At that time, I was envious and envious of the people in the group who seemed to have good relationships and chatted happily. "You can't confirm your friendship without talking, and if you have a real friendship, you'll know without talking," I often thought. From the discussion so far, we can see that we have to speak in order to understand the feelings of the other person. I changed my reaction, and the change in the reaction of the other person at that time constantly judged whether the other person's feelings and my feelings were the same. I see now.

With this remedy, you can read the air and understand the feelings of the other person. Regarding this, I will also practice it. I'll let you know if there is any progress.

5. The true nature of positive and negative thinking

Mathematically think about "I can't do it because I'm a negative thinker," which people who are victims of bullying and moral harassment tend to fall into.

It is mathematically thought that positive thinking and negative thinking are the difference between easy selection of positive things and easy selection of negative things when calculating emotion $x$ from equation (30). 
Now, I considered equations ( 5) and (15). The precondition at this time was to look at the good and bad things of the event and add the emotions before the emotions occurred. It turns out that it is a combinatorial problem, as we considered in equation (1). With that in mind, we can see that the number of events that receive negative emotions and the number of events that receive positive emotions are actually the same.

In other words, in fact, there are the same number of positives and negatives, but when you select from equation (30), you just choose one or the other. Things have both good and bad sides. Even if you tend to choose negative things, it's a good idea to keep in mind that there are the same number of positive and negative things, and to look at the positive side without thinking too much about it. I also want to avoid thinking too much about "why can I only take it negatively?"

6. Is there really a one-dimensional random walk?

The precondition so far is that emotion processing is performed by a one-dimensional random walk. In other words, it is assumed that you have selected positive emotions and negative emotions with a probability of $\frac{1}{2}$. Many people think that they are really doing it with a probability of $\frac{1}{2}$, or that they have a high probability of choosing either positive or negative.

So suppose the probability of choosing positive is $p$ instead of $\frac{1}{2}$. Then the probability of choosing a negative is $1-p$. At this time, the mean of the one-dimensional random walk is $n(2 p-1)$, and the variance is $4 n p(1-p)-n^{2}(2 p-1)^{2}$. The following equation is obtained by transforming the equation for variance.

$$
-4(1+n)\left(p-\frac{1}{2}\right)^{2}+1
$$

Since this value must be greater than or equal to 0 , the following conditions are obtained:

$$
\frac{1}{2}-\frac{1}{2 \sqrt{1+n}} \leq p \leq \frac{1}{2}+\frac{1}{2 \sqrt{1+n}}
$$

Therefore, the longer the emotion processing is performed, that is, when $n \rightarrow \infty$, the result is as follows.

$$
\frac{1}{2} \leq p \leq \frac{1}{2}
$$

In other words, like everyone who has done a long number of one-dimensional random walks, the probability must be $\frac{1}{2}$.

No, I haven't been bothered for a long time. In the first place, some people may say that $t \rightarrow 1$, that is, one second does not usually bother you. In Section 2.3, we showed that a 
discrete one-dimensional random walk is the same as a continuous Wiener process. The following relationships have been obtained between the one-dimensional random walk and the Wiener process. This is called Donsker's theorem. Here, $S_{\lfloor n t\rfloor}$ is the value obtained by randomly adding -1 and 1 for the maximum number of natural numbers up to $n t$.

$$
\lim _{n \rightarrow \infty} \frac{S_{\lfloor n t \mid}}{\sqrt{n}}=W_{t}, 0 \leq t \leq 1
$$

That is, for any short time between $0 \leq t \leq 1$, the one-dimensional random walk converges on the Wiener process. You can do $\infty$ times of one-dimensional random walks. You can choose $\infty$ times, and if you feel both positive and negative emotions, then equation ( 37 ) is satisfied.

In other words, in my model, a person who can feel both positive and negative emotions repeats a one-dimensional random walk with a probability of $\frac{1}{2}$. If the probability is not $\frac{1}{2}$, then the variance cannot be obtained, so statistical processing cannot be performed, and the probability density function of emotions with positive and negative emotions cannot be obtained. You can only have one of the emotions. If you can feel both emotions, you don't have to worry that you are different from a normal, neurotypical person from the probability density function of your emotions. You can't build relationships well only because you don't know how to read the air. I am also a person who can feel the same positive and negative emotions as normal, neurotypical, and has the same reaction function, the only difference is that I did not know how to read the air. I will try to think that it is.

\section{Conclusion}

We considered how human emotional processing is performed, and the probability density function and reaction function of emotions generated by emotional processing for events. As a result, it was found that the probability density function and the reaction function of the emotions of individuals and groups are expressed by the same equations ( 5 ) and ( 15), which is why the group is established. We also analyzed that the cause of bullying and moral harassment is that people who do not understand how to read the air are divided into those who attack and those who are attacked. I also thought mathematically about how to deal with people who are not familiar with their surroundings and who are suffering from bullying and power harassment. I don't get used to the surroundings well, and I'm bullied because I don't know how to read the air, so I'm floating because I'm reacting differently from the surroundings, and the bullying and moral harassment perpetrators make a reaction that I find interesting. I knew it 
was because of it. As a countermeasure, I also proposed how to read the air, as to what the ratio and maximum value of positive and negative emotions in the reaction function are. I will try it myself. I will report any progress.

It would be an unexpected joy if it helps. 